\title{
On enhanced tilt strategies for tilting trains
}

\author{
B. Kufver ${ }^{1} \&$ R. Persson ${ }^{2}$ \\ ${ }^{I}$ Ferroplan, Sweden \\ ${ }^{2}$ Bombardier, Sweden
}

\begin{abstract}
Many railways have taken tilting trains into operation on lines with horizontal curves with small radii. Tilting trains have vehicle bodies that can roll inwards, thus reducing the lateral acceleration perceived by the passengers. Hence, tilting trains can run through curves at an enhanced speed. However, too much tilt can cause motion sickness among sensitive passengers. The tilt motions, generated by computer-controlled actuators should be optimised with care, taking the local track geometry and actual train speed into account. This paper presents tilt algorithms aimed at balancing conflicting objectives. Furthermore, the paper discusses the usefulness of route files (track geometry data) onboard the train and possible positioning systems.
\end{abstract}

Keywords: tilting train, tilt algorithm, tilt strategy, alignment, cant, passenger comfort, motion sickness.

\section{Introduction}

The idea of tilting trains on lines with curves with small radii was discussed already during the 1930s [1,2]. Tilting trains have vehicle bodies that can roll inwards, thus reducing the lateral acceleration perceived by the passengers. Hence, tilting trains can run through curves at an enhanced speed. Nowadays, it is a mature technology that many railways have taken into revenue service.

However, experience shows that tilting trains can cause motion sickness among sensitive passengers [3-11]. The mechanisms behind motion sickness are not fully understood, but there is evidence that high roll velocities, generated by steep superelevation ramps in the tracks and by a high compensation ratio in the tilt system, contribute to the onset of motion sickness. Hence, too little tilting will cause discomfort caused by high lateral acceleration, while too much tilting may provoke motion sickness. 
Most existing tilting trains use a fixed compensation ratio (i.e. the amount of tilt is proportional to the lateral acceleration). This paper presents an enhanced approach, where the compensation ratio depends on local track conditions and the train speed.

\section{Vehicle motions on curves}

\subsection{Vertical curves}

On circular curves in the vertical alignment, there will be an additional vertical acceleration which should be superimposed on the gravitational acceleration. The additional vertical acceleration depends on the vehicle speed to the power of two, and therefore, is higher for a tilting train running at an enhanced speed.

\subsection{Circular curves in the horizontal alignment}

When the vehicle is running on circular curves in the horizontal alignment, there will be a horizontal acceleration which depends on the vehicle speed to the power of two. By arranging cant and using a tilt system, the vehicle body will roll inwards at an angle $\vartheta$. In the inclined vehicle body, the perceived lateral $a_{L}$ and vertical accelerations $a_{V}$ change according to eqns (1) and (2).

$$
\begin{aligned}
& a_{L}=\frac{V^{2}}{R} \cdot \cos (\vartheta)-\left(g+\frac{V^{2}}{R_{v}}\right) \cdot \sin (\vartheta) \\
& a_{V}=\frac{V^{2}}{R} \cdot \sin (\vartheta)+\left(g+\frac{V^{2}}{R_{v}}\right) \cdot \cos (\vartheta)
\end{aligned}
$$

where $V$ is vehicle speed in $\mathrm{m} / \mathrm{s}, R$ is radius of horizontal curve in metres, $\vartheta$ is total roll angle (depends on cant, suspension characteristics of the vehicle and tilt system), and $R_{v}$ is radius of vertical curve in metres.

A reduction of $a_{L}$ by an increased roll angle $\vartheta$ is always correlated with increased roll motions and increased vertical acceleration $a_{V}$. (Perceived yaw and pitch motions are also affected by the roll angle, but these effects are not in focus in this paper.)

\subsection{Transition curves in the horizontal alignment}

Transition curves are track segments where horizontal curvature changes gradually between straight tracks and circular curves (and between circular curves of different radii). It is normal practice to arrange superelevation ramps at the same location as the transition curves. It is also normal practice to apply the tilt motions on the transition curves, with no intentional delays. 
Motion quantities of particular interest are maximum values for lateral acceleration, lateral jerk (the rate of change of lateral acceleration), roll angle and roll velocity (the rate of change of roll angle). Figures 1-3 show different cases with respect to phase relations between the tilt motion and the track alignment.

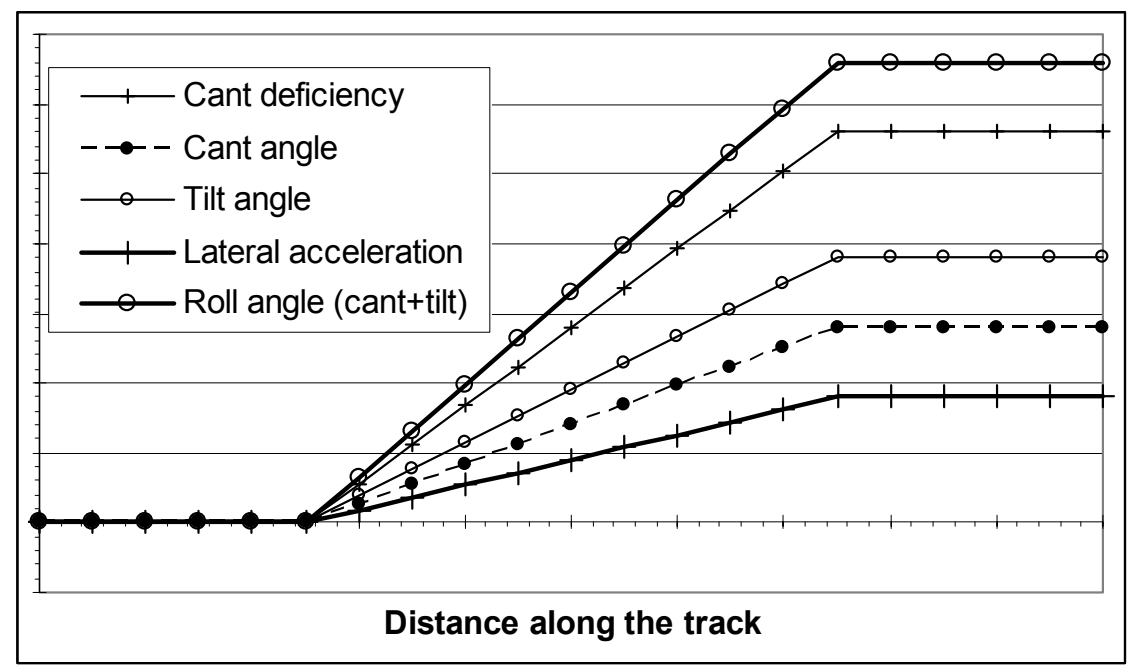

Figure 1: Entry transition: Motion quantities when the tilt motion is in phase with the alignment.

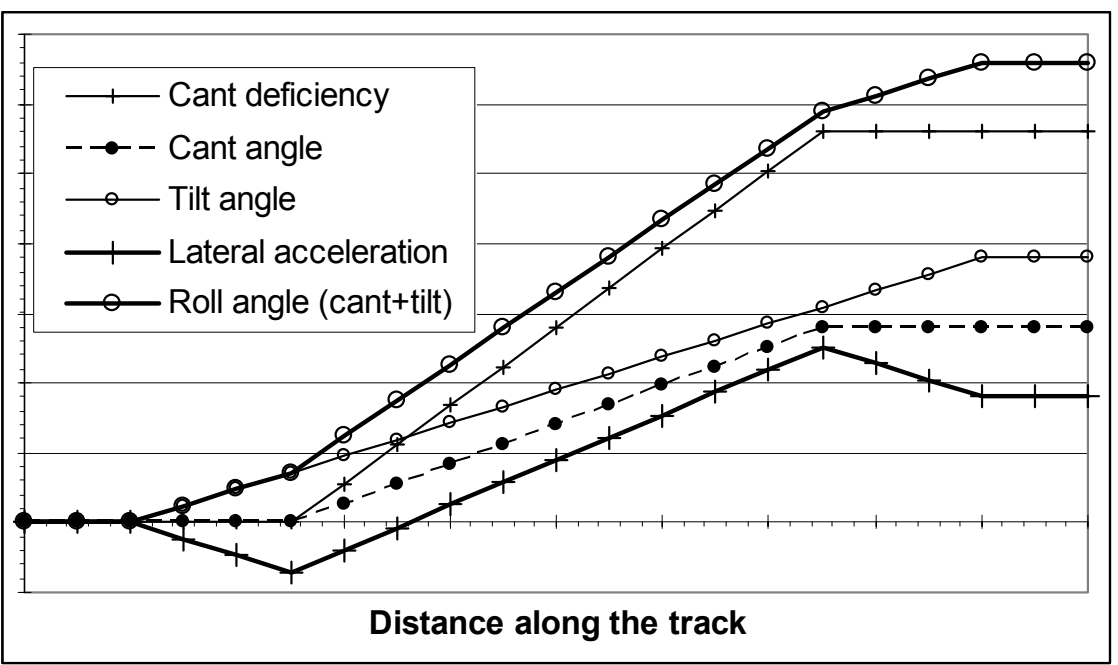

Figure 2: Entry transition: Motion quantities when the tilt motion is lengthened compared to the length of the transition curve. 


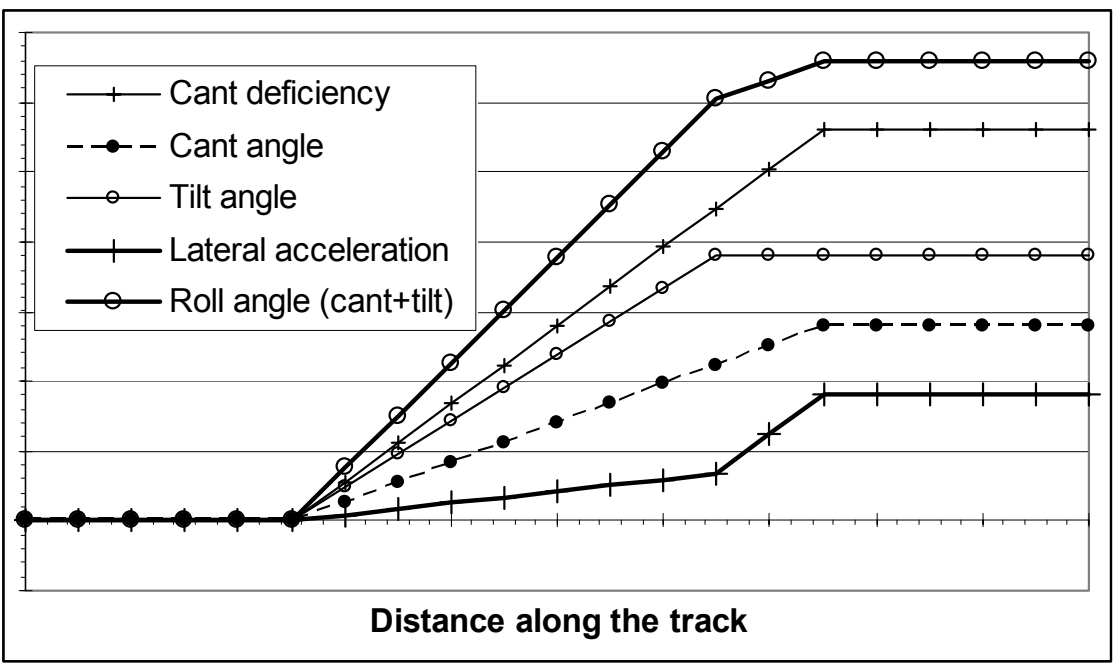

Figure 3: Entry transition: Motion quantities when the tilt angle saturates before the vehicle enters the circular curve.

In Figure 2, it can be seen that a longer tilt transition than the curve transition reduces the roll velocity (the rate of change of roll angle) but increases the maximum lateral jerk (the rate of change of lateral acceleration). If the mismatch between geometry and tilt occurs at the end of the entry transition, there will be a local maximum in the lateral acceleration, while if the mismatch occurs at the beginning of the entry transition there will be a change of direction of the perceived lateral acceleration. Current knowledge does not prove whether or not this is an advantage, but it seems reasonable to assume that a possible lengthening of the tilt motion should not be very pronounced.

In Figure 3, both maximum roll velocity and maximum lateral jerk are higher than in Figure 1 where the motions are in phase. This situation may occur with a fixed compensation ratio, which is too high for curves with very high lateral acceleration in the track plane. Reducing the tilt compensation ratio would be advantageous in this case, so that maximum tilt angle is reached at the end of the transition and not earlier. However, a general reduction may add discomfort on curves with less lateral acceleration in the track plane.

Transition curves with S-shaped curvature functions and S-shaped superelevation ramps have been investigated and vehicle response on these kind of alignments have not proven to be better than on traditional transition curves with a linear change of curvature and cant [12-14].

The present paper focuses on motion patterns according to Figure 1, but the tilt strategy is applicable also to cases according to Figure 2. An advanced tilt strategy should preclude cases according to Figure 3. 


\section{Comfort criteria}

Comfort disturbances due to high-speed curving are of two major categories:

1. Instantaneous discomfort when horizontal curves are entered.

2. Motion sickness, which is believed to be an accumulated effect of curving.

\subsection{Passenger comfort on curve transitions}

From extensive tests with tilting and non-tilting trains in United Kingdom [5], $P_{C T}$ functions for (instantaneous) passenger comfort on curve transitions have been derived as:

for standing passengers

$$
P_{C T}=\max (28.54 \cdot \ddot{y}+20.69 \cdot \dddot{y}-11.1,0)+0.185 \cdot(\dot{\vartheta})^{2.283}
$$

and for seated passengers

$$
P_{C T}=\max (8.97 \cdot \ddot{y}+9.68 \cdot \dddot{y}-5.9,0)+0.120 \cdot(\dot{\vartheta})^{1.626}
$$

where $\ddot{y}$ is maximum magnitude of lateral acceleration in $\mathrm{m} / \mathrm{s}^{2}, \dddot{y}$ is maximum magnitude of lateral jerk in $\mathrm{m} / \mathrm{s}^{3}$, and $\dot{\vartheta}$ is maximum magnitude of roll velocity in degrees/s. (For low-pass filtering and other evaluation procedures, see [15].)

The $P_{C T}$ functions express the expected percentage of dissatisfied passengers when the vehicle is running on transition curves where the lateral acceleration increases. They have been included in a European prestandard [15] of comfort evaluation.

\subsection{Motion sickness}

Even though motion sickness is a rare phenomenon in railway traffic, it has been reported from France [9], Great Britain [3, 5, 11], Italy [3], Japan [3, 4, 6], Sweden $[7,10]$ and Switzerland [8] that tilting trains sometimes cause motion sickness. Motion sickness is probably not correlated to a single curve but is rather an accumulated effect from several curves. According to ORE [3], the Japanese railways believe the problems to be associated with short transition curves. In Japan, limits of 5 degrees $/ \mathrm{s}$ for roll velocity and 15 degrees $/ \mathrm{s}^{2}$ for roll acceleration have been introduced, and the problems with motion sickness have decreased, but are not totally eliminated [6].

Swedish research in this area uses net dose models where motion doses (such as integrated squared roll velocity) are accumulated over time, and where a leakage component can quantify the recovery from nausea when the vehicle is running over more straight sections of the track [16, 17]. Roll motions are believed to be a major contributor to motion sickness [10], even though vertical, lateral, yaw and pitch motions may contribute as well. 


\section{Optimisation algorithms}

In the $P_{C T}$ functions, eqns (3) and (4), the first term (the lateral term) is zero for low values of lateral acceleration and lateral jerk, while the second term (the roll term) is zero only for a constant roll angle $(\dot{\vartheta}=0)$.

The analysis below assumes that comfort disturbances are minimised either for standing or seated passengers.

\subsection{Minimising $\mathbf{P}_{\mathrm{CT}}$}

For an entry transition, between a straight and a circular curve, the following cases may occur. In all cases, the train needs to "know" in advance the radius, cant and transition length (as well as the train speed).

Case Ia: There is no cant on the curve, and the lateral term is zero also for $\vartheta=0$. Lowest $P_{C T}$ is achieved if the tilt motion is switched off (or just compensates for outward sway due to primary and secondary suspensions). This is believed to be advantageous also with respect to motion sickness since it eliminates contributions to the doses of roll motions.

Case Ib: There is cant on the curve, but the lateral term is zero also for $\vartheta=0$. Lowest $P_{C T}$ is achieved if the tilt motion eliminates the cant angle. As in Case Ia, this is an advantage also for motion sickness.

Case II: A roll angle is required to reduce the lateral term to zero. The smallest possible roll angle is applied to reduce the lateral term to zero and to minimise contributions to doses of roll motions. The maximum tilt angle which the train can provide is not exceeded. A predefined limit for roll velocity (such as 5 degrees per second) is not exceeded. By combining eqns (1), (3) and (4), the optimal roll angle for standing and seated passengers can be calculated (see eqns (5) and (6) respectively).

$$
\begin{aligned}
& \left(\frac{V^{2}}{R} \cdot \cos (\vartheta)-\left(g+\frac{V^{2}}{R_{v}}\right) \cdot \sin (\vartheta)\right) \cdot\left(28.54+\frac{20.69 \cdot V}{L_{t}}\right)=11.1 \\
& \left(\frac{V^{2}}{R} \cdot \cos (\vartheta)-\left(g+\frac{V^{2}}{R_{v}}\right) \cdot \sin (\vartheta)\right) \cdot\left(8.97+\frac{9.68 \cdot V}{L_{t}}\right)=5.9
\end{aligned}
$$

where $L_{t}$ is the length of the transition curve in metres.

When the optimal roll angle has been calculated, the cant angle should be subtracted and the rate of change of tilt angle can be calculated using the values of the train speed and length of the transition curve.

Case III: If the tilt angle derived in Case II exceeds the maximum tilt angle for the actual type of train, the calculation of the rate of change of tilt angle is based on this maximum tilt angle. This eliminates the risk of the saturation of the 
tilt system according to Figure 3, and reduces maximum values for both lateral jerk and roll velocity.

Case IV: If the tilt angle derived in Case II or Case III generates a roll velocity (on the entry transition or the run-out transition) greater than the predefined limit, the angle is reduced to meet this requirement. This will increase the resulting $P_{C T}$ values (increase the instantaneous discomfort), but will reduce the tendencies to motion sickness.

When these rules are applied for calculating the amount of tilt, the tilt compensation ratio will vary from curve to curve. $P_{C T}$ will be minimised, maintaining a boundary condition for roll velocities, based on experiences of tendencies to motion sickness reported from Japan.

\subsection{Reducing the doses of the roll motions further}

If the predicted accumulated dose of roll motions is still too high, when the strategy in Section 4.1 is applied, there are two strategies to reduce the dose.

Strategy A reduces the limit for the roll velocity below the value used to define Case IV curves. This may reduce the amount of tilt on curves with high $P_{C T}$ values, which then would cause even higher instantaneous discomfort.

Strategy B reduces the amount of roll motions on curves where $P_{C T}$ values are zero or very low. Strategy B primarily affects the Case Ia, Ib and II curves. Instead of the target $P_{C T} \approx 0$, a new target is defined (such as $P_{C T}=3 \%$ ). The target may be adjusted until a sufficiently small dose of roll motions is predicted.

Eqns (7) and (8), for standing and seated passengers respectively, defines the roll angle $\vartheta$ for Case II curves with target $P_{C T}=T \%$.

$$
\begin{gathered}
\left(\frac{V^{2}}{R} \cdot \cos (\vartheta)-\left(g+\frac{V^{2}}{R_{v}}\right) \cdot \sin (\vartheta)\right) \cdot\left(28.54+\frac{20.69 \cdot V}{L_{t}}\right) \\
-11.1+0.185 \cdot\left(\frac{V \cdot \vartheta}{L_{t}}\right)^{2.283}=T \\
\left(\frac{V^{2}}{R} \cdot \cos (\vartheta)-\left(g+\frac{V^{2}}{R_{v}}\right) \cdot \sin (\vartheta)\right) \cdot\left(8.97+\frac{9.68 \cdot V}{L_{t}}\right) \\
-5.9+0.120 \cdot\left(\frac{V \cdot \vartheta}{L_{t}}\right)^{1.626}=T
\end{gathered}
$$

When these rules are applied for reducing the doses of roll, the tilt will be reduced on the curves with the best comfort and maintained at higher values for curves where the lateral term in the $P_{C T}$ functions generates most discomfort. 


\subsection{Numerical examples}

These numerical examples assume that the vehicle speed is $180 \mathrm{~km} / \mathrm{h}(V=50 \mathrm{~m} / \mathrm{s})$ and that the tilt system saturates at a net tilt angle of 6.5 degrees. This net angle includes the outward sway of the vehicle body due to the primary and secondary suspensions.

The horizontal curves are defined in Table 1. The curves are not claimed to be representative. They are chosen just to illustrate the effect of the different tilt strategies. When doses of roll motions (degrees ${ }^{2} /$ second) are calculated, the entry and exit transitions for each curve are assumed to be of the same length, and no leakage is applied (the five curves may be assumed to be close to each other).

Table 1: $\quad$ Track data in the numerical examples.

\begin{tabular}{|c|c|c|c|}
\hline Curve number & Radius $(\mathrm{m})$ & Cant $(\mathrm{mm})$ & Transition curve $(\mathrm{m})$ \\
\hline 1 & 2540 & 90 & 50 \\
\hline 2 & 1500 & 20 & 80 \\
\hline 3 & 1150 & 100 & 120 \\
\hline 4 & 850 & 150 & 200 \\
\hline 5 & 850 & 150 & 118 \\
\hline
\end{tabular}

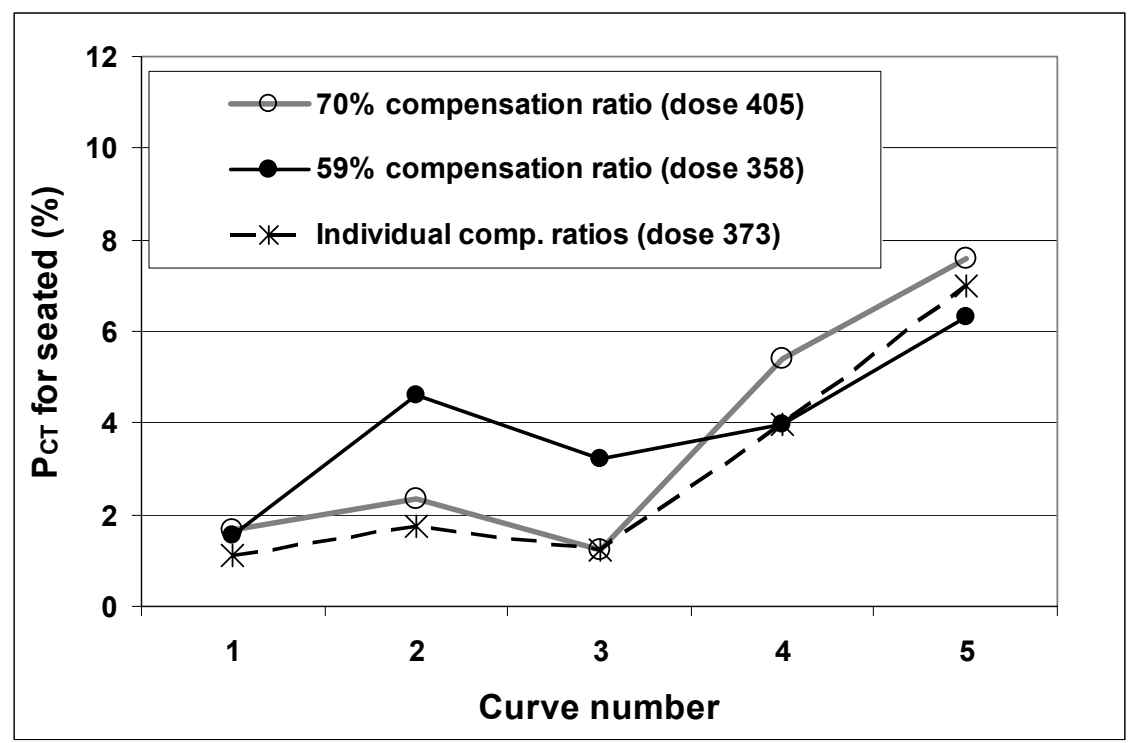

Figure 4: $\quad P_{C T}$ and doses of roll motions in the numerical examples.

A constant compensation ratio of $70 \%$ gives a saturation of the tilt on the transitions to curves 4 and 5 . A lower ratio of $59 \%$ reduces $P_{C T}$ on these two curves, but increases $P_{C T}$ on curves 2 and 3, see Figure 4 . With an individual 
choice of compensation ratios, the $P_{C T}$ values can be optimised for each curve, taking into account a limit of 5 degrees per second for the roll velocity (which is binding for curve number 5). If the resulting dose of roll velocity of $373 \mathrm{deg}^{2} / \mathrm{s}$ is considered too high, a reduction of roll motions according to Strategy A will increase $P_{C T}$ on curve 5, while Strategy B will increase $P_{C T}$ on curves 1-3.

\section{Data and computer aspects}

The tilt strategies described in this paper require onboard data of the track geometry (such as curve radii, cant, lengths of transition curves and longitudinal distance between all tangent points). The data can either be the acquired from the track engineer's register of track geometry, or by motion measurements by the tilting train itself. If the train measures lateral acceleration, roll velocity and train speed, then the necessary track geometry data can be calculated and stored on the onboard computer for use on future runs.

When the train has the route data, it needs to know its position along the track. The position can be determined from a measurement of longitudinal distance, which can be calibrated by known positions of signalling transponders, GPS-measurements and/or autocorrelation with the route data.

\section{Discussion and conclusions}

The tilt system of a tilting train reduces the perceived lateral acceleration and lateral jerk, but increases the roll motions (among others). Therefore the tilt motion generated by computer-controlled actuators should be optimised with care, taking local track geometry and actual train speed into account.

The tilt control system that is discussed here has the following advantages. The optimal tilt angle can be calculated and arranged, taking local track characteristics into account. If it is found advantageous, the tilt motion may be started slightly before the transition curve. Unintentional tilt motions, caused by track irregularities, can be eliminated.

Furthermore, delays in the tilt motion for the first vehicle can be eliminated and the maximum tilt angle can be taken into consideration at the beginning of the transition curve (eliminating the of risk saturation of the tilt system).

In case the doses of roll motions are predicted to be too high, the amount of tilt can be reduced where instantaneous comfort on curve entries anyway is good. High values for tilt motions are maintained where the lateral motions may cause the most discomfort.

\section{Acknowledgement}

The authors thank Bombardier for permission to publish this paper. 


\section{References}

[1] Deischl, WVV. Linienverbesserungen oder gesteuerte Achsen? Verkehrstechnische Woche, 31(9), pp. 97-108, 1938.

[2] Drechsel, A. Die Lösung der Schnellverkehrsfrage durch den kurvneigenden Kreiselwagen. Zeitung des Vereins Mitteleuropeischer Eisenbahnverwaltungen, 78(20)-78(21), pp. 377-385, 397-403, 1938.

[3] ORE. Body tilt coaches - Operational experience with body tilt coaches (BT), ORE S1037 Rp1, ORE: Utrecht, 1985.

[4] Koyanagi, S. Ride quality evaluation of a pendulum car. Quarterly Report of RTRI, 26(3), pp. 89-92, 1985.

[5] Harborough, P.R. Passenger comfort during high speed curving - analysis and conclusions, BRR TR DOS 017, British Rail Research: Derby, 1986.

[6] Ohno, H. What aspect is needed for a better understanding of tilt sickness? Quarterly Report of RTRI, 37(1), pp. 9-13, 1996.

[7] Förstberg, J. \& Ledin, T. Discomfort caused by low-frequency motions - A literature survey of hypotheses and possible causes of motion sickness. VTI Meddelande 802A, VTI: Linköping, 1996.

[8] Hughes, M. Tilt nausea is bad business. Railway Gazette International, 153(4), p. 249, 1997.

[9] Cleon, et al. Research on motion sickness. Proc. of World Congress on Railway Research 1999, Tokyo.

[10] Förstberg, J. Ride comfort and motion sickness in tilting trains - Human responses to motion environments in train and simulator experiments. PhD thesis, TRITA-FKT Report 2000:28, KTH: Stockholm.

[11] Brume, M. Why are Pendolinos so nauseating? Rail Professional, 93, p. 5, 2004.

[12] Kufver, B. Realigning railways in track renewals - Linear versus S-shaped superelevation ramps. Proc. of Railway Engineering -99, ECS Publications: Edinburgh. ISBN 0-947644-39-3.

[13] Kufver, B. Dynamic vehicle reactions on the Ruch type of S-shaped superelevation ramps. Proc. of Comprail 2000, CMP/WIT Press: Southampton, pp. 663-672. ISBN 1- 85312-598-9.

[14] Kufver, B. Optimisation of horizontal alignments for railways Procedures involving evaluation of dynamic vehicle response. $\mathrm{PhD}$ thesis, TRITA-FKT Report 2000:47, KTH: Stockholm.

[15] CEN. Railway applications - Ride comfort for passengers - Measurement and control. ENV 12299:1999, CEN: Brussels, 1999.

[16] Kufver, B. \& Förstberg, J. A net dose model for development of nausea. Proc. of $34^{\text {th }}$ meeting of the UK Group on Human Response to Vibration, Dunton, Essex, England, 1999.

[17] Kufver, B \& Förstberg, J. Research on the specific aspects of tilting. Proc. of World Congress on Railway Research 2001, Köln. 